\title{
Galactic acceleration phenomena
}

\author{
Yves A. Gallant \\ Laboratoire de Physique Théorique et Astroparticules, UMR 5027, CNRS/IN2P3, \\ Université Montpellier II, 34095 Montpellier Cedex 5, France \\ email: gallant@lpta.in2p3.fr
}

\begin{abstract}
I review the current status of our observational knowledge of prominent classes of particle accelerators in the Galaxy, namely shell-type supernova remnants (SNRs) and pulsar wind nebulae. I highlight in particular the contribution of the recent improvement in sensitivity of very-high-energy (VHE) $\gamma$-ray observations, which are currently the most direct probe of particle acceleration in the Galaxy up to energies of several hundreds of $\mathrm{TeV}$.

Shell-type SNRs have long been proposed as sources of the Galactic cosmic rays. In recent years, X-ray observations have revealed very thin, non-thermal rims in many young SNRs, and I discuss the implications of these observations for magnetic field amplification and the maximum particle energy attainable by acceleration at the blast wave. I then review the current status of the evidence for accelerated nuclei in these objects, and summarise current uncertainties.

The most numerous class of identified Galactic VHE gamma-ray sources is currently that of pulsar wind nebulae (PWNe). The emission from these objects is generally assumed to be predominantly leptonic, and I outline the new information provided by VHE gamma-ray observations beyond what could be inferred from observations of synchrotron emission.
\end{abstract}

Keywords. acceleration of particles, cosmic rays, gamma rays: observations, supernova remnants, shock waves, magnetic fields, pulsars: general, radiation mechanisms: nonthermal

\section{Galactic cosmic rays and VHE $\gamma$-rays}

Galactic cosmic rays (GCRs) were known to fill the Galaxy from EGRET observations of diffuse high-energy $\gamma$-ray emission. The recent discovery of diffuse very-high-energy (VHE) $\gamma$-ray emission (with energy $E_{\gamma}>100 \mathrm{GeV}$ ) from the Galactic Centre ridge by the HESS experiment (Aharonian et al. 2006a) illustrates the potential of this higher energy regime to close in on the sources of the GCRs. The spectrum of this VHE emission, thought to be hadronic in nature, is harder than the generic GCR spectrum, betraying the presence of recently accelerated cosmic rays.

\section{Shell-type supernova remnants}

Shell-type supernova remnants (SNRs) are widely believed to be the sources of the GCRs, due to energetics and composition arguments, and because of observational evidence for particle acceleration at their outer blast waves. In particular, the non-thermal $\mathrm{X}$-ray emission seen in most young SNRs is generally thought to be synchrotron emission from accelerated electrons. Recent X-ray observations have revealed this emission to originate in narrow rims in many young SNRs (see, e.g. Warren et al. 2005), which has been interpreted as evidence for turbulent magnetic field amplification at the shock. Such amplification would increase the maximum energy attainable by ions accelerated at this shock, but this still appears insufficient to reach the 'knee' of the GCR spectrum (Parizot et al. 2006). Indirect evidence for the efficient acceleration of ions is provided by SNR hydrodynamics, in particular the relative radii of the blast wave and contact discontinuity in some young SNRs (Decourchelle, Ellison \& Ballet 2000). 
VHE $\gamma$-ray observations have the potential to yield direct evidence for GCR acceleration in SNRs. Two shell-type SNRs have been detected and studied in great morphological and spectral detail by HESS, namely G 347.3-0.5 (or RX J1713.7-3946, Aharonian et al. 2006b) and G 266.2-1.2 (or RX J0852.0-4622). Both remnants emit dominantly non-thermal X-rays, with which the $\gamma$-ray emission shows a high degree of morphological correlation, and have relatively hard $\gamma$-ray spectra, with power-law indices $\Gamma \approx 2.25$. It is nonetheless still unclear whether this $\gamma$-ray emission is hadronic, from $\pi^{0}$ decay, or leptonic, via the inverse Compton (IC) process. The spectrum would tend to favour the former hypothesis, but the latter more naturally explains the morphological correspondence with X-rays.

At least four of the new VHE sources discovered in the HESS survey of the Galactic plane (Aharonian et al. 2006c) show convincing positional coincidences with SNRs, namely HESS J1640-465 (with G 338.3-0.0), J1713-381 (CTB 37B), J1834-087 (W41), and J1813-178 (G 12.8-0.0). In some of these SNRs, however, the VHE emission could originate in a yet unconfirmed central plerion (see below) rather than in the shell. More detailed, multi-wavelength studies should help clarify the nature of the VHE $\gamma$-ray emission in these SNRs.

\section{Pulsar wind nebulae}

In pulsar wind nebulae (PWNe), also known as plerionic SNRs, accelerated particles are thought to originate in the relativistic wind of a central pulsar. The observed nonthermal emission is usually assumed to be predominantly leptonic, consisting of synchrotron emission from radio waves to X-rays and beyond, and IC scattering in VHE $\gamma$-rays. As the target photon density is in general almost uniform on the scale of the PWN, VHE $\gamma$ rays then directly trace the spatial distribution of energetic electrons, unlike synchrotron emission which may also reflect magnetic field variations. The electron spectrum can also be directly inferred from the observed IC emission, provided the target photon density is known (Aharonian et al. 2006d; Porter, Moskalenko \& Strong 2006).

VHE $\gamma$-ray emission has been detected by HESS from seven established PWNe: the Crab Nebula, MSH 15-52, G0.9+0.1, Vela X, two sources in the Kookaburra, and the nebula of PSR B1823-13. In this last case, $\gamma$-ray spectral steepening away from the pulsar supports the identification of HESS J1825-137 as the PWN (Aharonian et al. 2007). In several of these sources, the centroid of the PWN appears significantly offset from the pulsar, possibly due to the passage of an asymmetric reverse shock. Several other sources discovered in the HESS survey of the Galactic plane may also be associated with energetic pulsars (Aharonian et al. 2006c). VHE $\gamma$-rays are thus opening a new and promising observational window on particle acceleration in PWNe.

\section{References}

Aharonian, F., Akhperjanian, A. G., Bazer-Bachi, A. R., et al. 2006a, Nature, 439, 695

Aharonian, F., Akhperjanian, A. G., Bazer-Bachi, A. R., et al. 2006b, A\&SA, 449, 223

Aharonian, F., Akhperjanian, A. G., Bazer-Bachi, A. R., et al. 2006c, ApJ, 636, 777

Aharonian, F., Akhperjanian, A. G., Bazer-Bachi, A. R., et al. 2006d, A\&SA (Letters), 448, L43

Aharonian, F., Akhperjanian, A. G., Bazer-Bachi, A. R., et al. 2007, A\&A, 661, 236

Decourchelle, A., Ellison, D. C., \& Ballet, J. 2000, ApJ (Letters), 543, L57

Parizot, E., Marcowith, A., Ballet, J., \& Gallant, Y. A. 2006, A\& $A, 453,387$

Porter, T. A., Moskalenko, I. V., \& Strong, A. W. 2006, ApJ (Letters), 648, L29

Warren, J. S., Hughes, J. P, Badenes, C., Ghavamian, P., McKee, C. F., Moffett, D., Plucinsky, P. P., Rakowski, C., Reynoso, E., \& Slane, P. 2005, ApJ, 634, 376 